\title{
High Priority Returned Lunar Samples
}

A White Paper submitted to the Planetary Science Decadal Survey 2023-2032

\author{
Primary Author \\ Sarah N. Valencia \\ University of Maryland College Park/NASA Goddard Space Flight Center \\ sarah.n.valencia@nasa.gov
}

\section{Co-Authors}

Natalie Curran, Catholic University of America/NASA Goddard Space Flight Center Jessica Flahaut, CRPG, CNRS/Université de Lorraine, France Juliane Gross, Rutgers University, NJ

Cameron M. Mercer, Catholic University of America/ /NASA Goddard Space Flight Center Daniel P. Moriarty III, University of Maryland College Park/NASA Goddard Space Flight Center

Clive R. Neal, University of Notre Dame, IN

Noah E. Petro, NASA Goddard Space Flight Center

Tabb C. Prissel, Lunar \& Planetary Institute/Universities Space Research Association Ryan N. Watkins, Planetary Science Institute

Wajiha Iqbal, Institut für Planetologie, Westfälische Wilhelms-Universität Münster

\author{
Signatories \\ Kris Zacny, Honeybee Robotics \\ Timothy Glotch, Stony Brook University \\ Trevor Graff, Jacobs/NASA Johnson Space Center \\ Kerri Donaldson Hanna, University of Central Florida \\ Jose M. Hurtado, Jr., The University of Texas at El Paso \\ R. Aileen Yingst, Planetary Science Institute \\ Joshua Cahill, Johns Hopkins Applied Physics Laboratory \\ Csilla Orgel, European Space Agency
}


The Apollo and Luna landing missions, as well as orbital remote sensing missions, have shaped our understanding of the origin and petrogenetic evolution of the Moon. Through a wide range of science investigations, the identification of new lithologies, the global mapping of the chemical heterogeneity of the lunar crust, the estimate of gravity anomalies, and measurements of surface temperature, a wide range of data and data sets are available for studying and understanding lunar processes and the shared Earth-Moon formation history. However, some important questions can only be addressed by detailed geochemical and physical investigations in laboratories on Earth on rock samples returned from the Moon. The return of new, strategically planned, lunar samples - through either robotic or human missions - are critically important for the future of lunar science, and would have an immediate, profound impact on our understanding of the Moon in the next decade.

\section{Overview of the Lunar Sample Collection}

The lunar sample collection consists of samples returned from the surface of the Moon during the Apollo (382 kg) and Luna missions (326 g), and from meteorites delivered to Earth after excavation during impacts to the lunar surface $(666 \mathrm{~kg}$; Meteoritical Bulletin as of July 4, 2020). Together, these samples provide a view of a Moon with diverse chemistry and complex geological processes. But, both the current returned sample collection and the lunar meteorites lack the necessary information to answer some of the most pressing outstanding questions about the evolution of the Moon. The Apollo and Luna samples were taken from within the Procellarum KREEP Terrane (PKT), a distinct chemical region on the Moon characterized by an enrichment in incompatible elements including potassium $(\mathrm{K})$, rare earth elements (REE), and phosphorus $(\mathrm{P})$. Thus, these samples are limited in both the geographic and geochemical range they represent and do not include examples of all lithologies known to occur on the Moon. While meteorites are currently the best samples to investigate areas other than the Apollo and Luna landing sites, rocks with known geographical provenance on the Moon are imperative to fully interpret how these samples fit into the formation and evolution of the Moon, and to link remote observations to sample information, which maximizes science return $[1,2]$.

\section{The Value of Additional Returned Samples from the Moon}

The Need for Additional Samples - Samples have not been returned from the Moon since the 1970s. Since that time, our understanding of the formation and evolution of the Moon has drastically improved, particularly considering data from modern remote sensing missions that was not available when sampling locations were chosen in the 1960s and 1970s. The Apollo mission locations were chosen to minimize mission risk, and owing to little understanding of the complexities of lunar chemistry and geology, science was not prioritized. With decades of both sample and remote sensing data in our repertoire, the lunar science community understands well what samples are now needed to continue to advance the field of lunar science. New samples are needed of lithologies that we have in the existing sample collection (e.g., mare basalts), but we need new samples taken in situ to correlate with, and maximize the science return of, remote observations, and because the samples we currently have are not representative of the full range of lunar compositions. Additionally, examples of never-before-sampled rocks (e.g., polar volatiles) are needed to close strategic knowledge gaps (SKGs) and answer current science questions. 
The Value of Sample Return vs. In Situ Analysis - In situ analysis of rocks on the Moon and other planetary bodies is undeniably scientifically valuable, and such measurements have yielded impressive results. However, in situ analyses do not have the capabilities or analytical precision available on Earth for the study of geological materials. Instrumentation on spacecraft operate in extreme environments and do not have advanced sample preparation capabilities. But, on Earth technically challenging measurements can be made owing to the relatively stable environment that allows for highly sensitive instrumentation, as well as specialized sample preparation (e.g., coating and sectioning). Another benefit of sample return is the ability to preserve sample material for future studies as science questions evolve or analytical techniques are improved and invented. The Apollo collection has had $>3200$ sample requests over the last 50 years, is still actively studied today, and stands as a testament to the lasting scientific value of returned samples $[3,4]$.

Types of Samples Needed in Earthbound Laboratories - While all samples returned from the Moon are precious and scientifically valuable, some specific types of samples are needed to fill existing SKGs and answer outstanding science questions. In addition to the soil samples and rocks that can be brought back in varying masses and diameters, deep drill cores are needed. Sampling by drilling allows for the investigation of samples shielded from the solar wind, as well as variations in chemistry with depth, thereby providing information on the bombardment history of the inner solar system, and the history of space weathering on the lunar surface.

Perhaps the most technologically challenging sample return is that of frozen samples from permanently shadowed regions (PSRs). Understanding the nature of volatiles in PSRs is critically important for the future of human exploration and inhabitation on the Moon (see below, and Prem et al., 2020 white paper). However, the sampling, transportation, storage, and analyses of these frozen samples is one of the highest priority challenges we must overcome to prepare for the future of humans living and working on the Moon, and thus, needs significant investment of time and funding for technology development in the coming decade [e.g., 5,6].

Benefit Beyond the Moon - Sample return from the lunar surface has benefits far beyond learning about the formation and evolution of the Moon. Without water, plate tectonics, or an atmosphere, the lunar surface preserves the record of its evolution since the solidification of the lunar magma ocean (LMO). Thus, the Moon is a record of over 4 billion years of inner solar system history. Examination of lunar samples can answer outstanding questions about the history of the Sun, the formation of evolved magma on a 1-plate body, and the impact history of the inner solar system, among many other things. Additionally, owing to its relative accessibility, the Moon can serve as a test bed for perfecting sample return technology in preparation for sample return missions to Mars, Mercury, other moons, asteroids, etc.

\section{High Priority Sample Types for Future Sample Return Missions}

Listed below are some of the most critical types of samples that are needed for physical and chemical examination in laboratories on Earth. This list is based on consensus built through publications [e.g., 1, 3, 7] and community-built documents, e.g., the Lunar Science for Landed Missions workshop report [5], which recorded locations where these rock types can be collected.

Silicic (Felsic) Rocks- Non-basaltic volcanism is rare on the Moon [8]. Rare granite or its finegrained equivalent, felsite (rhyolite), and more mafic alkali norite or alkali gabbro (monzogabbro) were found as small rocks and clasts, mainly in Apollo 12, 14, and 15 samples [e.g., 9]. Further 
evidence of both intrusive and extrusive silicic rocks on the Moon was brought by decades of remote sensing observations. Spectroscopically unique features (i.e., red spots) that are characterized by deep UV absorptions, have long been studied as possible pre-mare/non-mare volcanism [e.g., 9]. Recent analyses suggest that five red spots-Hansteen Alpha, the Gruithuisen Domes, the Lassell Massif, Aristarchus crater, and the Compton Belkovich Volcanic Complex (CBVC) - were derived from evolved magmas [10-12]. Whereas the Gruithuisen Domes, Hansteen Alpha, and the CBVC spectral anomalies are associated with steep-sided volcanic domes, and are therefore indicative of extrusive silicic volcanism [e.g., 12,13], silicic rocks exposed by impact craters in the Aristarchus and Lassell regions suggest excavation of silicic plutons formed at depth [e.g., 11]. The magmatic processes capable of producing the observed highly evolved compositions are poorly understood: silicate/ liquid immiscibility, basaltic underplating, and fractional crystallization of late stage magmas from the LMO have all been invoked [e.g., 14], illustrating the need to return samples from both intrusive and extrusive silicic locations.

Mantle Materials - Currently, there are no confirmed mantle materials in the lunar sample collection. Consequently, our understanding of the mantle (and its role in shaping surface features and interior properties) is derived primarily from geophysical observations, laboratory experiments, and petrological analyses of crustal materials, volcanic glasses, and mare basalts. In particular, our simplified understanding of mantle stratigraphy is shaped by numerical and experimental simulations of LMO crystallization [15-20]. The leading paradigm for mantle evolution is the cumulate mantle overturn hypothesis [e.g., 21-23], whereby dense ilmenitebearing cumulates sink into the lunar interior and less-dense olivine-rich material buoyantly rises and invades the upper mantle. Contrary to the current paradigms, very little of the upper lunar mantle appears to be dominated by olivine [24]. Thus, there is no consensus on what lithologies comprise the relevant upper mantle strata. Several candidate lithologies have been proposed based on LMO crystallization models and remote sensing observations: (1) dunite, (2) low-Ca pyroxenite, and (3) ilmenite-bearing cumulates (rich in Ca,Fe-pyroxenes and KREEP). At face value, the arguments for these different upper mantle compositions seem at odds. However, the evolution of the lunar mantle during and after LMO crystallization unfolded over hundreds of millions of years. Thus, it is reasonable that impact structures of different sizes, ages, and locations would have exposed upper mantle materials with varying compositions.

Several of the largest basins are estimated to have excavated and melted mantle materials from beneath the $\sim 40 \mathrm{~km}$ thick lunar crust. The South Pole - Aitken Basin (SPA) is the largest lunar impact structure, and therefore the most likely to have excavated abundant mantle materials. Recent analyses have demonstrated that ejecta in the NW quadrant of the basin bear the compositional signatures (Ca,Fe-rich pyroxenes; elevated Fe, KREEP, and Ti) expected in upper mantle during the final stages of LMO crystallization [25]. As such, it is the most unambiguous exposure of mantle materials on the lunar surface, and may have been exposed during the final stages of mantle evolution. Sampling these materials is critical for understanding the timing and petrology of magma ocean crystallization and subsequent lunar evolution.

Farside Highlands - The lunar surface represents an archive of information that preserved the early history and evolution of the Moon, and of the solar system. The first Ferroan anorthosites (FAN) were found in the returned Apollo 11 samples and were identified as a key lunar lithology. Examination of FAN led to the simple view of the evolution of the Moon, in which it crystallized from an LMO [26]. Since this time, investigations of anorthositic rocks have allowed for continued testing of the LMO hypothesis and have demonstrated a more complex geologic history of our 
Moon than that previously provided by a simple LMO model. Most lunar anorthositic meteorites, and clasts within, have different compositions from those of the majority of returned Apollo samples in both major and trace elements. New investigations of FAN clasts in Apollo 15 and 16 samples support these findings and show that these samples (meteoritic anorthosites and some Apollo FAN) cannot be explained by crystallization from a single magma composition, leading to new questions about LMO evolution [27, 28]. Additionally, orbital remote sensing data revealed a nearside-farside compositional dichotomy with the farside of the Moon being more Mg-rich than the nearside FAN lithologies [29, 30]. Further, outcrops of pure anorthosite (PAN) with $<2-3$ vol.\% mafic minerals have been detected globally, associated with basins and large craters, suggesting PAN occurs largely at a depth range of $3-30 \mathrm{~km}[31,32]$. PAN could potentially be the result of extremely efficient density-driven separation of plagioclase from pyroxene and Fe-rich residual LMO melt [33]. Therefore, anorthosite outcrops from the farside terrains (both Mg-rich anorthosites and PAN) are good sample-return landing sites as these samples will help us to understand and place better constraints on the LMO formation and crystallization, including the models of crustal formation and evolution that can be extrapolated to other planetary bodies, and subsequently lunar igneous evolution.

Non-Mare, Non-Silicic Volcanic Products - A number of unusual volcanic emplacements have been observed across the lunar surface, exhibiting anomalous compositions and ages. For instance, in SPA, a $\sim 650 \mathrm{~km}$ resurfacing deposit known as the SPA Compositional Anomaly (SPACA) [34] exhibits a distinct non-mare mineralogy, and is thought to be a volcanic emplacement influenced by the unique geophysical environment within the Moon's largest impact structure. Mons Marguerite (formerly known as Mafic Mound [34]) is a volcanic construct in the center of SPACA and exhibits similar mineralogical properties, representing an ideal target to sample this unusual material. Other unusual volcanic emplacements on the lunar surface include the irregular mare patches, which appear extremely young, which would have implications for the duration of magmatism on the Moon [e.g., 35]. These unusual materials must be understood to fully capture the diverse behavior, timing, and thermal evolution of the lunar interior.

Spinel-rich Rocks - Although spinel is an accessory mineral in most igneous rocks, its presence and composition can be used to constrain melt compositions and thus better understand the formation of rock suites and the composition of mantle sources [e.g., 36-38]. Only small amounts of Mg-spinel are found in lunar samples, but in 2010, outcrop-scale areas in the lunar highlands that are rich in Mg-spinel were detected with the Moon Mineralogy Mapper $\left(\mathrm{M}^{3}\right)$ on the Chandrayaan-1 spacecraft. These pink-spinel-anorthosites (PSA) occur as distinct localized rock types in anorthositic highland settings [39] and exhibit no other detectable mafic minerals. To date, at least $23 \mathrm{Mg}$-spinel-rich sites have been identified globally including locations within Mare Moscoviense, the PKT (e.g., Albategnius, Copernicus, Goodacre), Feldspathic Highlands (e.g., Kovalevskaya, Lowell), and South Pole - Aitken basin (e.g., Hausen, McKellar). All individual exposures of $\mathrm{Mg}$-spinel anorthosite are relatively small, typically a few hundred meters in extent, although regional clusters exist [40,41]. Most of the locations have been detected in the central peaks of craters, although the location sites also include dispersed areas in terraced walls of a few large feldspathic craters, two areas of hypothesized non-mare volcanism, and along the inner ring of four major basins in areas of relatively low crustal thickness, and thus appears to be originally associated with a lower crust origin, perhaps predating the basin-forming period. Remote compositional results and experimental investigations indicate that $\mathrm{Mg}$-spinel could be common in the lower crust and suggest that crust-melt interactions (endogenic processes) are likely the 
mechanism of Mg-spinel formation. Returned samples of PSA would help to better constrain the origin and petrogenesis of this potentially common rock type of the lower crust, and provide new insight into the thermal evolution of the lunar crust.

Impact Melt Rocks - Impact cratering is a fundamental geologic process that has affected the evolution of all solid bodies in the Solar System. The impact record archived on the Moon spans most of Solar System history [e.g., 42,43] and has become the lens through which the bombardment histories of other planetary bodies are interpreted, including those of Earth and Mars. However, questions still remain regarding important aspects of the Moon's bombardment history (e.g., lunar cataclysm). For example, the onset, duration, and intensity of impacts during the basin-forming epoch are still widely debated [44-45], and the matter is complicated by potential oversampling of the Imbrium impact event from Apollo samples [e.g., 46]. Impact melt rock samples returned from both the oldest and youngest basins would provide a unique opportunity to test the extent and duration of lunar cataclysm. The age of returned samples from the oldest (SPA) and youngest (Orientale and Schrodinger) basins, as well as intermediate-aged basins (e.g., Nectaris and Crisium) would anchor the lunar basin-forming epoch.

The intermediate and recent cratering record is also critical to determining if the impact flux has remained constant through time. This relates to the variability in Solar System dynamics (e.g., the breakup of asteroids) and effects such things as mass extinctions, the rise of life, and the current impact hazards to Earth. The Moon has been undisturbed from many of the geological processes that affected and removed the impact record on the Earth, and so provides a more preserved archive of this record. Sampling primary impact craters such as Copernicus (0.80 - 1.50 b.y) and Tycho ( $\sim 0.10$ b.y) would provide important calibration points for the lunar chronology at young ages. Quantifying the timing, intensity, and source of materials impacting the Moon will improve our understanding of: the dynamical evolution of impactor populations in the inner Solar System; the geologic evolution of planetary surfaces; the timing of the delivery of volatiles and organic compounds; and the potential effects of impacts on emerging biological systems.

Mare Basalts - Mare basalts are a window into the thermal and magmatic history of the Moon. Basalts were collected at all of the Apollo sites, and exhibit ages between 3.10-3.90 b.y., with a few basalt clasts in Apollo 14 breccias being 4.20 b.y. Crater count studies of different nearside mare terrains [47] show a wider array of ages. Crater size-frequency distribution data has shown that the P60 basalt unit, located south of the Aristarchus Plateau, has a model age of $\sim 1$ b.y [48]. In spite of this, our current temporal knowledge of magmatic processes on the Moon are represented by lunar meteorites, which represent the oldest ( 4.35 b.y, Kalahari 009) [49] and youngest (e.g., 2.93 b.y., North-West Africa (NWA) 032) [50] known basalts from the different lunar collections. Recent work on lunar meteorites has also added to the growing evidence that basaltic volcanism started on the Moon as early as $\sim 4.35$ b.y $[51,52]$. These oldest samples are may be from volcanic deposits that have been obscured by impact ejecta, known as cryptomare $[49,52]$, which occur widely across the Moon [53]. However, although lunar meteorites may be from regions that were not sampled by Apollo, these samples lack the same geological context as their source location is unknown. Returning samples from areas of cryptomare or young basalts (e.g., the P60 unit) would provide an anchor to bracket mare basaltic volcanism.

Furthermore, the Apollo missions only collected samples from the nearside equatorial region of the Moon, an area dominated by the PKT. However, mare basalts can be found on farside and regions remote from the Apollo landing sites. These are found within craters within SPA basin, Mare Moscoviense, Tsiolkovskiy crater, and Mare Australe. Sample return from younger and 
older basaltic plains would not only help in understanding the thermal evolution of and mantle heterogeneity within the Moon, but would also better constrain the crater count curve that is used to constrain the ages of planetary surfaces within the inner Solar System.

Pyroclastic Deposits - Pyroclastic deposits are largely glass beads erupted from deep in the lunar mantle and thus record the chemistry of their source regions. Large and regional pyroclastic deposits $\left(>1000 \mathrm{~km}^{2}\right)$ have been mapped and characterized across the lunar surface [e.g., 54,55]. These pyroclastic deposits seem to have different modes of origin that result in distinct modes of occurrence. The sampled Apollo 15 and 17 volcanic glasses are associated with regional deposits and are some of the most primitive mantle melts in the sample collection. Hydration has been detected from orbit in many of these regional deposits and could be linked to the mantle-derived water in these volcanic glasses [56]. No additional pyroclastic glass beads other than the Apollo 15 and 17 samples have been found (in returned or meteoritic samples). Pyroclastic samples returned from locations other than Apollo 15 and 17 could help answer questions about mantle composition, mantle derived water and other volatiles. For example, Sinus Aestuum is a regional dark pyroclastic deposit that exhibits distinct and abundant Fe- or Cr-rich spinel [e.g., 57] and would be an ideal target to study both pyroclastic deposits and spinel rich samples.

Polar Volatiles - In the time since the last decadal survey was written, new remote sensing lunar missions and increased analytical capabilities for lunar samples has transformed our understanding of the types and concentrations of volatiles on the Moon. However, many important questions remain about the lunar volatile cycle (discussed in detail in [6]). Understanding the volatile cycle on the Moon has the potential to be enabling for a sustained human presence on the lunar surface. Of particular interest is the potential for surficial water that has been identified by remote spacecraft [e.g., 58]. This surficial water, along with other volatile compounds appear to be trapped, near-surface, in PSRs near the north and south poles of the Moon [e.g., 59]. Returning samples taken from PSRs, and particularly drill samples, will enable the study of volatile production and transport on the Moon which will close SKGs and significantly advance our ability to have a sustained human presence on the Moon.

\section{Recommendations}

Recommendation \#1: Sample return must be prioritized in the next 10 years of lunar science and exploration. Just as the Apollo samples have fueled our scientific knowledge of the Moon for the past 50 years, new samples will answer outstanding scientific questions and continue to fuel lunar science for decades to come.

Recommendation \#2: It is critical that the planetary science community fosters an interdisciplinary, diverse, equitable, inclusive, and accessible environment. As demonstrated in this report, lunar sample return requires drawing on perspectives spanning the gamut of planetary science, geoscience, technology, engineering, and beyond. We strongly encourage the decadal survey to consider the state of the profession and the issues of equity, diversity, inclusion, and accessibility — not as separable issues, but as critical steps on the pathway to understanding the Moon and the entire solar system. Background information on the current lack of diversity in our community and specific, actionable, and practical recommendations can be found in [60-63].

\section{References}

[1] Tartèse et al. (2019) Space Sci. Rev. 215, 54. [2] Joy \& Arai (2013) Astro. \& Geophys. 54, 4. [3] Pernet-Fisher et al. (2019) Astro. \& Geophys. 60, 4.22-4.28. [4] Zeigler et al. (2019) Elements 
15, 286-287. [5] Jawin et al. (2019) Earth \& Space Sci. 6, 2-40. [6] Prem et al. (2020) Lunar Volatiles and Solar System Science, White Paper. [7] Shearer \& Borg (2006) Geochem. 66, 163185. [8] Valencia \& Watkins et al. (2020) End-member volcanism in the absence of plate tectonics: silicic volcanism on the Moon, White Paper [9] Marvin, et al. (1991) Proc. Lunar Planet. Sci. 21, 119-135. [10] Hagerty et al. (2006) JGR: Planets, 111(E6). [11] Glotch et al. (2010) Science 329, 1510-1513. [12] Jolliff et al. (2011) Nature Geosci. 4, 566-571. [13] Hawke et al. (2003) JGR, 108(E7), 5069. [14] Seddio et al. (2013) Am. Min. 98, 1697-1713. [15] Snyder et al. (1992) Geochim. Cosmochim. Acta 56, 3809-3823. [16] Elardo et al. (2011) Geochim. Cosmochim. Acta 75, 3024-3045. [17] Elkins-Tanton et al. (2011) Earth and Planet. Sci. Lett. 304, 326-336. [18] Lin et al. (2017) Earth and Planet. Sci. Lett. 471, 104-116. [19] Charlier et al. (2018) Geochim. Cosmochim. Acta 234, 50-69. [20] Rapp \& Draper (2018) Meteor. and Planet. Sci. 53, 1432-1455. [21] Hess \& Parmentier (1995) Earth Planet. Sci. Lett. 134, 501-514. [22] Zhang et al. (2017) Geophys. Res. Lett. 44, 6543-6552. [23] Li et al. (2019) JGR Planets, doi: 10.1029/2018JE005905. [24] Melosh (2017) Geology 45, 1063-1066. [25] Moriarty (2020) JGR Planets, (Submitted) [26] Wood et al. (1970) Science 167, 602-604. [27] Gross et al. (2014) Earth Planet Sci. Lett. 388, 318328. [28] Russell et al. (2014) Phil. Trans.of the Royal Society A: Math., Phys. and Engin. Sci. 372, 20130241. [29] Arai et al. (2008) Earth, Planets and Space 60, 433-444. [30] Ohtake et al. (2012) Nat. Geosci. 5, 384-388. [31] Ohtake et al. (2009) Nature 461, 236-U110. [32] Donaldson Hanna et al. (2014) JGR Planets 119, 1516-1545. [33] Dygert et al. (2017) Geophys. Res. Lett. 44. [34] Moriarty \& Pieters (2015) Geophys. Res. Lett. 42, 7907-7915. [35] Elder et al. (2017) Icarus 290, 224-237. [36] Gross \& Treiman (2011). JGR Planets 116. E10 [37] Prissel et al. (2014) Earth Planet Sci. Lett. 403, 144-156. [38] Prissel et al. (2016) Am. Min. 101, 1624-1635. [39] Pieters et al. (2011) JGR Planets 116. [40] Pieters et al. (2014) Am. Min. 99, 1893-1910. [41] Sun et al. (2017) Earth Planet Sci. Lett. 465, 48-58. [42] Wilhelms (1987). 1348. Washington D. C.: USGS [43] Speyerer et al. (2016) Nature 538, 215-218. [44] Cohen et al. (2000) Science 290, 1754-1756. [45] Morbidelli et al. (2018) Icarus 305, 262-276. [46] Zellner (2017) Origins of Life and Evolution of Biospheres 47, 261-280. [47] Hiesinger et al. (2011) USGS Special Paper 477. [48] Stadermann (2018) Icarus 309, 45-60. [49] Terada et al. (2007) Nature 450, 849-852. [50] Borg et al. (2009) Geochim. Cosmochim. Acta 73, 3963-3980. [51] Snape et al. (2018) Earth Planet Sci. Lett. 502, 84-95. [52] Curran et al. (2019) Met. \& Planet. Sci. 54, 1401-1430. [53] Whitten \& Head (2015) Icarus 247, 150-171. [54] Gaddis et al. (2003) Icarus 161, 262-280. [55] Gustafson et al. (2012) JGR Planets 117. [56] Milliken \& Li (2017) Nature Geosci. 10, 561-565. [57] Yamamoto et al. (2013) Geophys. Res. Lett. 40, 4549-4554. [58] Colaprete et al. (2010) Science 330, 463-468. [59] Li et al. (2018) 8907-8912. [60] Rivera-Valentín et al. (2020) Who is Missing in Planetary Science?: Demographics showing Black and Latinx scientists are the most underrepresented, White Paper. [61] Rathburn et al. (2020) Who is Missing in Planetary Science?: Recommendations to increase the number of Black and Latinx scientists, White Paper. [62] Strauss et al. (2020) Nonbinary inclusion in planetary science, White Paper. [63] Milazzo et al. (2020) DEIA 101: Why is diversity important?, White Paper. 